\title{
ABSTRACT
}

Meetings of The Keio Medical Society

\section{New Perspective on the Treatment of Intractable Gastrointestinal Cancers: Role of Combination Therapies}

\author{
Dan G. Duda \\ Massachusetts General Hospital, Boston, USA \\ Massachusetts General Hospital Research Institute, Boston, USA \\ Harvard Medical School, Boston, USA
}

Unresectable gastrointestinal cancers, such as gastric, hepatocellular, biliary tract or pancreatic carcinomas, are often resistant to anti-cancer systemic therapies, and often recur locally or even after aggressive local therapies leading to dismal survival rates. Recent developments in oncology, have offered renewed hoped for the development of more efficacious therapies. For example, our understanding of the oncogenic drivers in carcinogenesis has increased exponentially, and may potentially allow personalization of therapy. In addition, a great interest has been recently to target immune checkpoints, and unleash the potential of the immune system against cancers. Finally, new radiation techniques (proton therapy) allow delivery of curative doses in a safely manner. However, optimal translation of these new therapeutic approaches will require a concerted effort in clinical trials and in preclinical studies. I will present results from clinical correlative studies and preclinical models of these diseases performed at our institution and in collaboration with other American and European investigators. The insights gained from this bench-to-the-bedside and back approach raise the hope for a more efficient development of targeted agents in gastrointestinal cancers, with the goal of increasing survival in patients afflicted with these aggressive and deadly diseases.

(Presented at the 1951st Meeting, October 30th, 2017) 